\title{
Relative intensity calibration of CDS-GIS detectors on SOHO using a plasma diagnostic technique
}

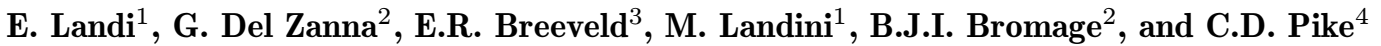 \\ 1 Department of Astronomy and Space Science, University of Florence, Italy \\ 2 Centre for Astrophysics, University of Central Lancashire, Preston, UK \\ 3 Mullard Space Science Laboratory, University College London, Holmbury St. Mary, Dorking, RH5 6NT, UK \\ ${ }^{4}$ Rutherford Appleton Laboratory, Chilton, Didcot, UK
}

Received June 16, accepted September 24, 1998

\begin{abstract}
The internal intensity calibration of the Coronal Diagnostic Spectrometer (CDS) - Grazing Incidence Spectrometer (GIS) is studied using the Arcetri diagnostic method. A large number of spectral lines observed by the four GIS detectors in solar active and quiet regions is analysed in order to determine the relative intensity calibration curve of the instrument.

The plasma diagnostic method developed in Arcetri allows the measurement of the correction factors to the preflight CDS - GIS internal intensity calibration curves and of the relative calibration between different detectors. No gross deviations from the pre-flight calibration are found. Also the GIS 3 and GIS 4 second order sensitivites are measured.

The problems of fixed patterning, ghosting, anomalous line profiles and widths are also discussed, and a list of lines suitable for further diagnostic studies with GIS is presented.
\end{abstract}

Key words: space vehicles: instruments - techniques: miscellaneous

\section{Introduction}

The Coronal Diagnostic Spectrometer (CDS) on SOHO consists of two instruments: a Grazing Incidence Spectrometer (GIS) producing astigmatic spectra; and a Normal Incidence Spectrometer (NIS) which can provide images of the solar field of view, dispersed over two wavelength ranges. Together, GIS and NIS cover most of the extreme ultraviolet wavelength range from $150 \AA$ to $785 \AA$ (Harrison et al. 1995). The GIS instrument has four detectors, with spectral ranges $151 \AA-221 \AA, 256 \AA$ -

Send offprint requests to: E. Landi
$341 \AA, 393 \AA-492 \AA$ and $659 \AA-785 \AA$, while NIS covers the ranges $308 \AA-379 \AA$ and $513 \AA-633 \AA$.

This spectral region is extremely rich in emission lines from a large number of highly ionized ions of the most abundant elements, providing a unique diagnostic tool for investigating the temperature, density and chemical composition of the solar transition region and corona. Moreover it provides a laboratory for testing atomic physics models and theoretical calculations of collision rates and transition probabilities for ions formed at chromospheric to coronal temperatures.

Intensity calibration is a fundamental requirement for many scientific uses of the spectrometer, but obtaining such information is not an easy undertaking in the extreme ultraviolet spectral range. A pre-flight calibration was performed (Bromage et al. 1996) using a limited number of emission lines, providing an initial measure of the mean sensitivity of each detector and a basis for absolute calibration of the instrument. Exposure to air between calibration and launch, as well as exposure to solar radiation in flight, will have modified the efficiency of the detectors in at least some regions. The large number of emission lines that are observed in the solar spectrum, coupled to the large amount of atomic data now available (CHIANTI - Dere et al. 1997, The Arcetri Spectral Code - Landi \& Landini 1998a, ADAS - Summers et al. 1996) and a new temperature and density diagnostic technique (Landi \& Landini 1997), allow a more detailed determination of the current (in-flight) relative intensity calibration.

This new diagnostic technique has been applied to GIS observations of a solar active region (AR) and of a quiet Sun region (QS), in order to derive correction factors which may be applied to the pre-flight intensity calibration of the instrument. This method has already been successfully applied to the CDS NIS spectrometer (Landi et al. 1997). 
Since the aim of this work is to assess the calibration of the GIS instrument, only a brief description of the emission lines used for this purpose is provided, leaving a more complete description of a full line list and of the diagnostic capabilities to a further paper. Together with the NIS quiet Sun spectral atlas (Brooks et al. 1998a), these line lists represents a spectral atlas for solar EUV line emission under both quiet Sun and active region conditions.

\section{The theoretical method}

\subsection{The L-function method for emission line analysis}

The method used for this calibration study is a byproduct of the temperature and density diagnostic procedure shown in Landi \& Landini (1997) and has already been applied by Landi et al. (1997) for determining the sensitivity of the NIS 1 instrument. As the adopted analysis method requires to determine the Differential Emission Measure (DEM) of the emitting plasma as a preliminary step, the $D E M$ diagnostic method described in Landi \& Landini (1997) has been adopted in the present study. The reader is referred to these two papers for details on the analysis method and on its application to calibration of EUV spectrometers.

Lines of the same ion which occur in more than one detector are used for intercalibration between these detectors and the second order lines available in the GIS 3 and 4 sections are used to evaluate the ratio of the second order to first order efficiency for those two detectors.

In the present study the $D E M$ functions are derived using lines coming from all the four GIS detectors, and therefore the determination of these curves provides a first indication of the relative intensity calibration of the spectral detectors.

For this kind of study high quality atomic data are required. In the present study the Arcetri Spectral Code (Landi \& Landini 1998a) is used; this is an update of the older version (Monsignori Fossi \& Landini 1995) using the whole CHIANTI database (Dere et al. 1997) and additional data for ions not yet included in CHIANTI. The ionization equilibrium of Arnaud \& Raymond (1992) has been adopted for the Iron ions, while the Arnaud \& Rothenflug (1985) ionization equilibrium data are used for the other elements. Ion fractions for the less abundant elements come instead from Landini \& Monsignori Fossi (1991). The element abundances are taken from Feldman (1992).

\section{The Grazing Incidence Spectrometer (GIS)}

The Grazing Incidence Spectrometer (GIS) consists of a grazing incidence spherical grating that disperses the incident light to four detectors placed along the Rowland Circle. It is astigmatic, focusing the image of the slit along

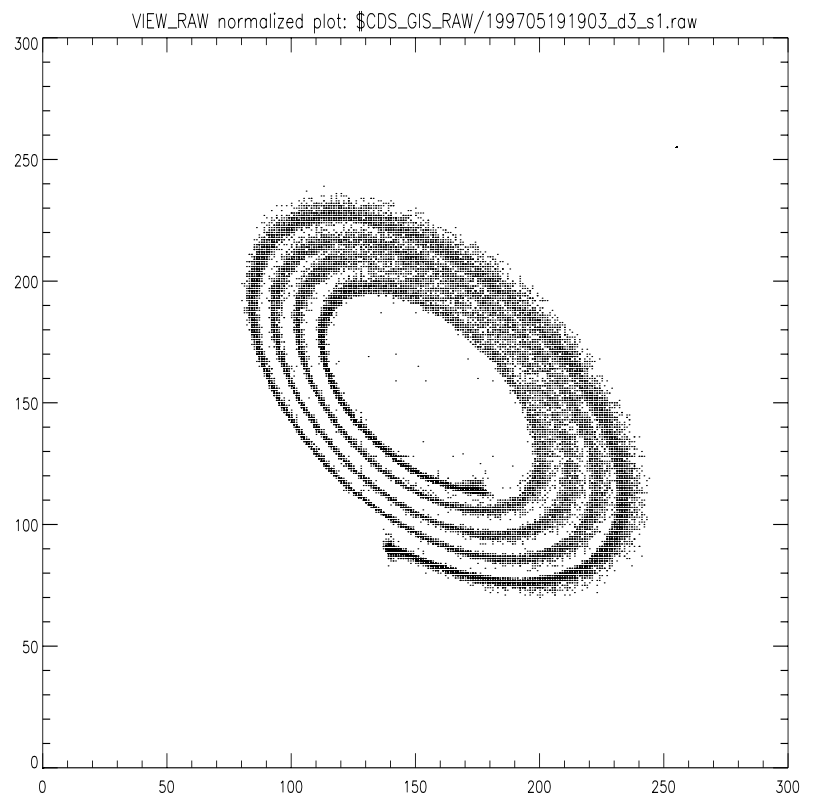

Fig. 1. Typical spiral pattern for the GIS detectors. The $x$ - and $y$-axis units are Analogue to Digital converter Units (ADU)

the direction of dispersion but not perpendicular to it. Images of the field of view may be obtained using a pinhole slit and rastering using movements of the slit and the scan mirror; normally, the raster covers the region of the Sun moving from south to north and from west to east. A more detailed description of the CDS instrument can be found in Harrison et al. (1995).

The GIS detectors are SPAN (spiral anode) MicroChannel Plate (MCP) detectors, described in detail in Breeveld et al. (1992) and Breeveld (1996). Raw data from the four detectors is collected on a regular basis to check the gain and spectral dispersion. The raw data consists of pairs of voltages produced in the spiral anode in response to each photon which falls on the detector over a period of time. These are plotted against each other to produce a spiral pattern, where the wavelength varies with distance along the spiral and the width of each arm is related to the noise in the electronics. A typical spiral pattern is displayed in Fig. 1. The number of counts at each radial position along the spiral is proportional to the number of photons hitting the front of the detector. The raw data is then used to produce four on-board look up tables (LUTs) which translate each voltage pair into a pixel position along the detectors, dependent on the wavelength. Because the gain in the micro channel plates is sensitive to the intensity, the voltage applied to the detector must be adjusted depending on the slit used and the solar conditions (e.g., active or quiet Sun) and a different LUT must be used in each case. The set of LUTs used in a given observation is identified by its GSET_ID.

There are various effects that must be taken into account before starting any analysis of GIS data, for example 
electronic dead time, short term gain depression and long term gain depression.

The GIS operates by sending a continuous stream of pixels to the Command and Data-Handling System (CDHS). During normal observations, counts on each pixel position are accumulated for the length of the exposure. An array of 2048 pixels is thus produced for each detector. The various electronic dead-times are such that any events occuring within about $10 \mu$ s will be rejected, and higher rates will render the detectors unusable.

Short term gain depression occurs when count rates are above 40. In this situation, the MCP cannot recharge fast enough to provide full gain for every event, and the data are not usable.

Long term gain depression is an ageing effect, a reduction in detector sensitivity over a long time interval due to continued illumination. The applied high voltage can be increased to compensate for the decrease in the MCP gain with time. However, the gain depression will be greatest at the position of the brightest lines so a wavelengthdependent effect will remain.

Other effects to be taken into account when analysing GIS spectra are fixed patterning and the problem of ghosts.

The profiles of the GIS lines are predominantly instrumental. They are corrupted by the superposition of a spiky effect on the whole spectrum, referred to as fixed patterning. This is caused by the finite number of bits employed in the electronics to obtain the detector positions (pixels). Counts falling near the boundary of a pixel can be shifted to neighbouring pixels, producing narrow spikes and troughs in the profiles of the emission lines. Weaker lines can be swamped by this effect. This process does not alter the total counts recorded in an emission line; it merely displaces some of them.

Ghosts result from the spiral nature of the SPAN detectors (see Fig. 1). In the case of some lines, the photon events will tend to spread across to the neighbouring arms of the spiral. This means that a portion of the counts belonging to a line can be shifted into different parts of the spectrum, giving rise to spurious spectral lines if they fall in a spectral region void of lines, or providing extra intensity to already existing spectral lines. They can also fall outside the spectral range of the detector.

Hereafter we will refer to a spectral line whose intensity is enhanced by the presence of counts coming from a line in a different arm of the spiral as a ghosted line, while the lines whose counts have been partially shifted toward other regions of the spectrum will be referred to as ghosting lines. The displaced counts will be called ghosts.

Each line can generate no more than two ghosts, one blue-shifted and the other red-shifted (see Fig. 1). Attempts are made to reduce the number of ghosts by a judicious choice of look-up tables. For each application of a set of GIS set-up parameters, ghosts will appear in the same positions. Once the applied LUT is known, it is therefore possible to deduce where ghosts will appear, and also which regions of the spectrum are unlikely to be affected by them.

\section{The observations}

In the present study two full spectral atlases are analyzed, one emitted by a solar active region, and the other in quiet Sun conditions. Both of them were obtained by rastering over a $30 \times 30 \operatorname{arcsec}^{2}$ area on the solar disk with 15 mirror positions and 15 slit positions using the $2^{\prime \prime} \times 2^{\prime \prime}$ slit. For each of the resulting 225 exposures of both sets of spectra the exposure time was $50 \mathrm{~s}$. The quiet Sun observation was obtained on September $2^{\text {nd }} 1996$; the corresponding data file is known as s4552. Its GSET_ID number is 41 . The active region spectrum (s4554) was taken immediately after the quiet Sun observation. Its GSET_ID is 47. The two datasets were taken close together in order to eliminate any possible long term time variation of the GIS intensity calibration between one observation and the other, so that the results obtained from the two spectra could be directly compared.

\section{Data reduction}

A program has been developed as a part of the CDS software which allows both raw data corrections and intensity calibration. Various corrections and checks have been applied to the spectra in order to calibrate them. The checks included dead time corrections and short term gain depression. Corrections for flat field and long term gain depression were not included since they are not yet available. It is known, however, that these corrections are very small.

The telescope effective area, as calculated from the preflight calibration (Bromage et al. 1996), has been used to convert the count rates to phot $\mathrm{cm}^{-2} \mathrm{~s}^{-1} \operatorname{arcsec}^{-2}$. This is shown in Table 5 . These values are the mean sensitivities over the wavelength range of each of the four detectors. Bromage et al. (1996) report that there was no evidence of wavelength-dependence for the sensitivity of each detector.

After applying the various correction factors, spatially averaged spectra were formed, in order to increase the signal to noise ratio. Monochromatic images of the rastered areas on the Sun were obtained by summing up the pixel intensities of the brightest lines. These were examined for inhomogeneity in the emitting regions, before spatially averaging the spectra. This is necessary because, as noted by Landi \& Landini (1997), the L-functions will cross each other only if the emitting plasma is homogeneous. In case there are inhomogeneities it is possible that some departures of the L-functions from the expected behaviour may occur due to lines emitted in different solar conditions, making it more difficult to determine possible intensity calibration problems. 
a

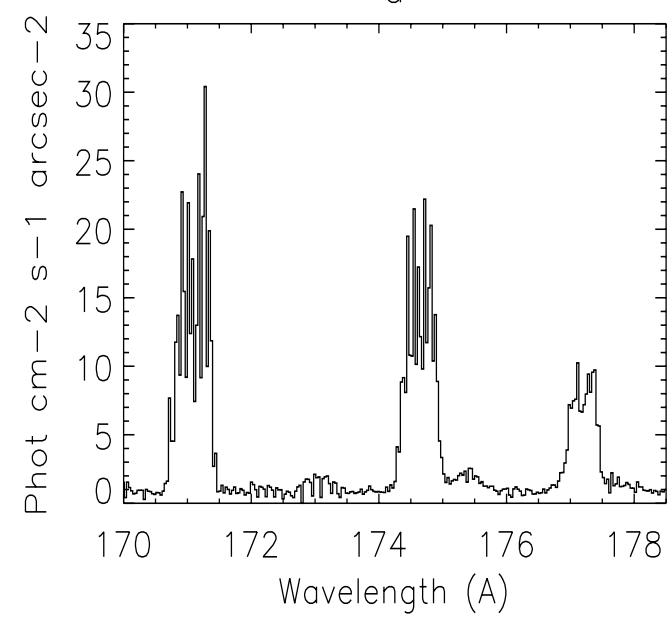

C

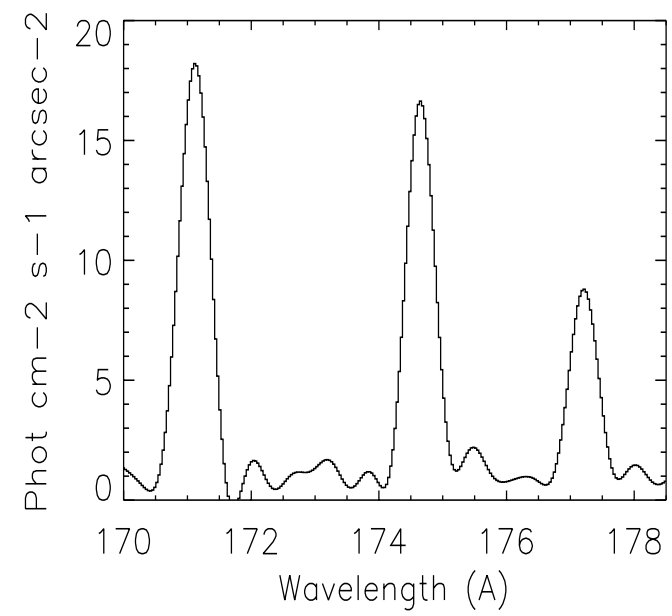

b

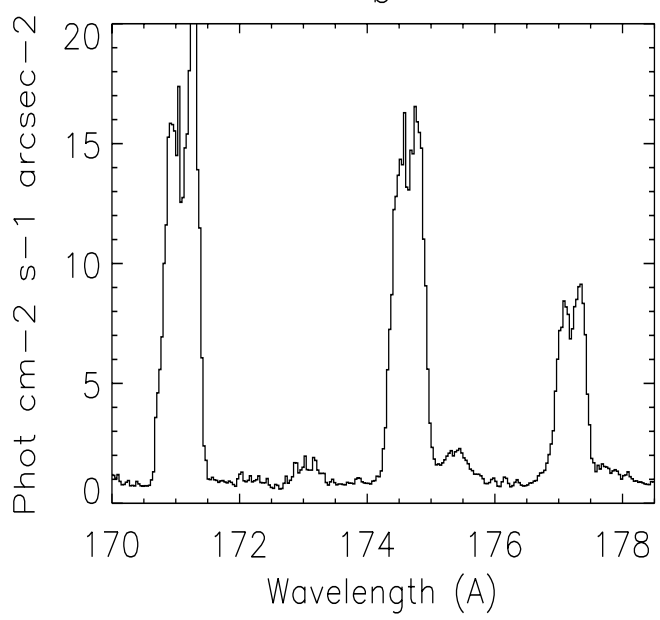

d

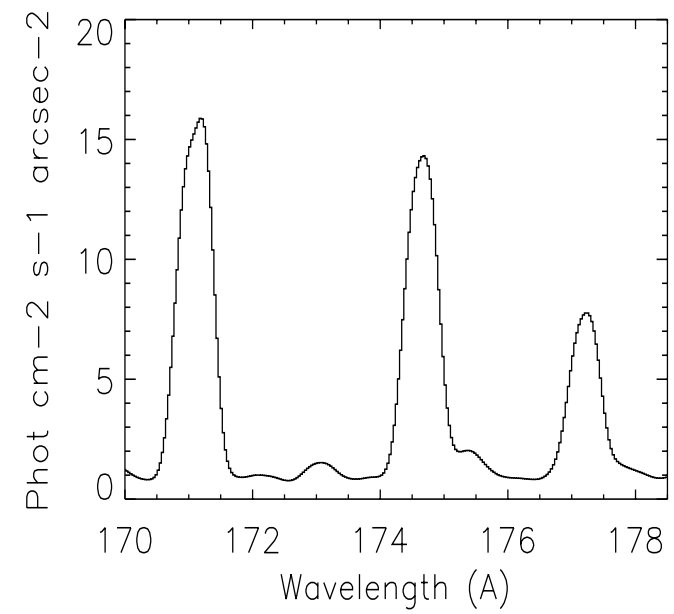

Fig. 2. Effects of different smoothing procedures applied to a portion of the GIS 1 active region spectrum. a) Original spectrum; b) Box-smoothed spectrum; c) Fourier-filtered spectrum; d) Gaussian-convolved spectrum

The emission appeared fairly uniform over the QS region, and there was no evidence of significant inhomogeneities, while the AR images showed large variations in the intensity in the hottest lines, revealing a very complex morphology, the structure varying with temperature. A map of the intensity ratio of two Fe XIII strongly densitysensitive lines $202.0 \AA / 203.8 \AA$ indicated considerable variations in electron density. As a result, only the area with ratio values between 1.2 and 1.4 was used to form an average AR spectrum. This selected region broadly covered the hottest part of the total emitting area, and had an electron density $\simeq 10^{9.4} \mathrm{~cm}^{-3}$.

\subsection{Fixed patterning and line profiles}

The fixed patterning effects are shown in Fig. 2. In this figure a portion of GIS 1 fixed-patterned spectrum is shown (Fig. 2a), together with the same spectral region smoothed with three different smoothing methods (Figs. 2b, c and d). It is possible to see in Fig. 2a that strong line profiles are heavily changed, making it very difficult to fit line profiles and calculate their intensity. The smoothed spectra reveal the presence of weaker lines that in the raw spectrum were not easily distinguishable from the background and so were difficult to fit. It is therefore necessary to apply some kind of correction in order to restore the original line profiles without altering the total intensity of the lines. This is particularly important in the case of unresolved blended lines. It is impossible to rebuild the original pixel intensities from the observed spectrum, but because the spectral lines seen on the detectors are many pixels wide, it is possible to use a simple smoothing technique, which does not change total count-rates.

Four different methods were compared: boxcar filtering with 3 pixel width; convolution with a Gaussian function with a 2 pixel-wide $\sigma$; Fourier filtering; 4-pixel Hanning smoothing. Checks were made to verify that these methods preserve the total number of counts. 
The widths of the boxcar and Hanning smoothing and the $\sigma$ of the Gaussian smoothing were chosen as optimum compromise between a reasonable cleaning of the spectrum and the smallest broadening of the line profiles.

Figure 2 displays the comparison between the original GIS 1 spectrum and the results of smoothing between $169 \AA$ and $176 \AA$. The shape of the spectrum is much improved by all three smoothing procedures.

Boxcar and Hanning smoothing give nearly identical results; they are not able to remove completely the effects of fixed patterning (Fig. 2b), though these are greatly reduced. Resulting spectral lines show double peaked line profiles, more evident for most of the GIS 1 lines and less pronounced in the lines observed in the other detectors.

Fourier filtering (Fig. 2c) produces nearly Gaussian line profiles and a very clean spectrum, but alters the background, making it very difficult to identify and fit weak lines.

The Gaussian convolution is able to clean line profiles (Fig. 2d), and the resulting lines are very close to Gaussians, with flat background.

In most cases it seems that only heavy smoothing is able to provide Gaussian profiles, at the price of significant loss of spectral resolution. Moreover boxcar and Hanning smoothing are able to produce Gaussian line profiles only if the adopted width is larger than 10 pixels. This width is large compared to the width of the fixed patterning spikes, suggesting that the observed double peaked line profile should not be ascribed to fixed patterning effects only but to the instrumental response. The origin of this double peaked line profile is probably an optical effect, from a small mis-alignment of the telescope, scan mirror, grating, and detectors.

Since it is important to understand the impact of the smoothing procedures on the final line intensities, the line profile fitting CFIT package (Haugan 1997), part of the CDS software, has been used on each of these four spectra in order to calculate line intensities, position, width and the value of the background. A large number of lines from the four detectors has been used for this comparison.

The agreement between the results obtained from the smoothed spectra is always better than $10 \%$. Only a few very weak lines show greater differences between the Fourier filtered spectrum and the others due to the strong background alteration of the former. Moreover there is good agreement between the results obtained from fitting smoothed spectra and those summing counts over the width of the line. The difference in intensity was found to be less than $5 \%$ in most cases. The intensities obtained by fitting the raw data may be up to $20 \%$ less than from simply summing the counts in the line, because the fit often follows dips due to fixed patternng within the true profile of the line. Fitting the data smoothed by any of these four methods (or summing counts) therefore gives a consistently better result.
No significant change is found in line positions, while Gaussian smoothing increases the line widths, thus reducing the GIS spectral resolution.

In the present work 3-point boxcar smoothing has been adopted since it represents the best compromise between adequate smoothing and minimum distortion of the true line shape.

Even with the more strongly double-peaked lines, Gaussian fitting after boxcar smoothing yielded intensities within $5 \%$ of those obtained from summing counts.

\subsection{Ghosts}

Figures 3 and 4 show the red- and blue-shifted spectrum for the GIS 1 detector together with the regions which are likely to be unaffected by the ghosting problem (dashed line in the bottom panel). The regions more likely are indicated by a bold line. It is currently not easy to calculate precisely the positions where ghosts occur, and the boundaries of the regions shown in Figs. 3 and 4 are currently uncertain.

Understanding where the ghosts of each line fall is important, since the shifted counts reduce its apparent intensity and often fall close to other spectral lines, enhancing their intensity. An example of this is reported in Fig. 4, where the $173-182.5 \AA$ spectrum is displayed. It is possible to see that the three relatively weak spectral features observed in the red-shifted spectrum are ghosts of the Fex 174.5 $\AA$, Fe X 177.2 $\AA$ and Fe XI 180.4 $\AA$; in the blue-shifted spectrum the ghosting affects three other lines: Fe XII 192.4 А, Fe XII 195.1 $\AA$ and the Fe XIII 197.4 Only when a ghost appears in a region of the spectrum free of lines, is it possible to correct the ghosting line from which it came, while it is usually very difficult to distinguish between the ghost and any real lines with which it is blended.

One more complication is that ghosted lines are often themselves the source of ghosts, causing considerable problems in the analysis of the spectra. For example the Fe XII 192.4 $\AA$ line is probably ghosting the Fe X $174.5 \AA$, increasing the confusion in this spectral range.

Two methods are proposed to help in locating the ghosts more precisely. Once several non-ghosted and isolated lines have been identified with confidence, it is possible to use the diagnostic technique to identify a ghost. The ghost will enhance the intensity of the ghosted line, resulting in an L-function which is higher than expected. The contribution due to the ghost can then be found and traced back to its source line, and both the ghosting and ghosted lines can then be corrected. Progressing in this way, the spectrum may be reconstructed. However, the main limitation of this method is that the effect of ghosts can be confused with those of atomic physics and intensity calibration uncertainties, both of which cause the Lfunction values to be higher than expected. For this reason great caution is required in such a study. The other 


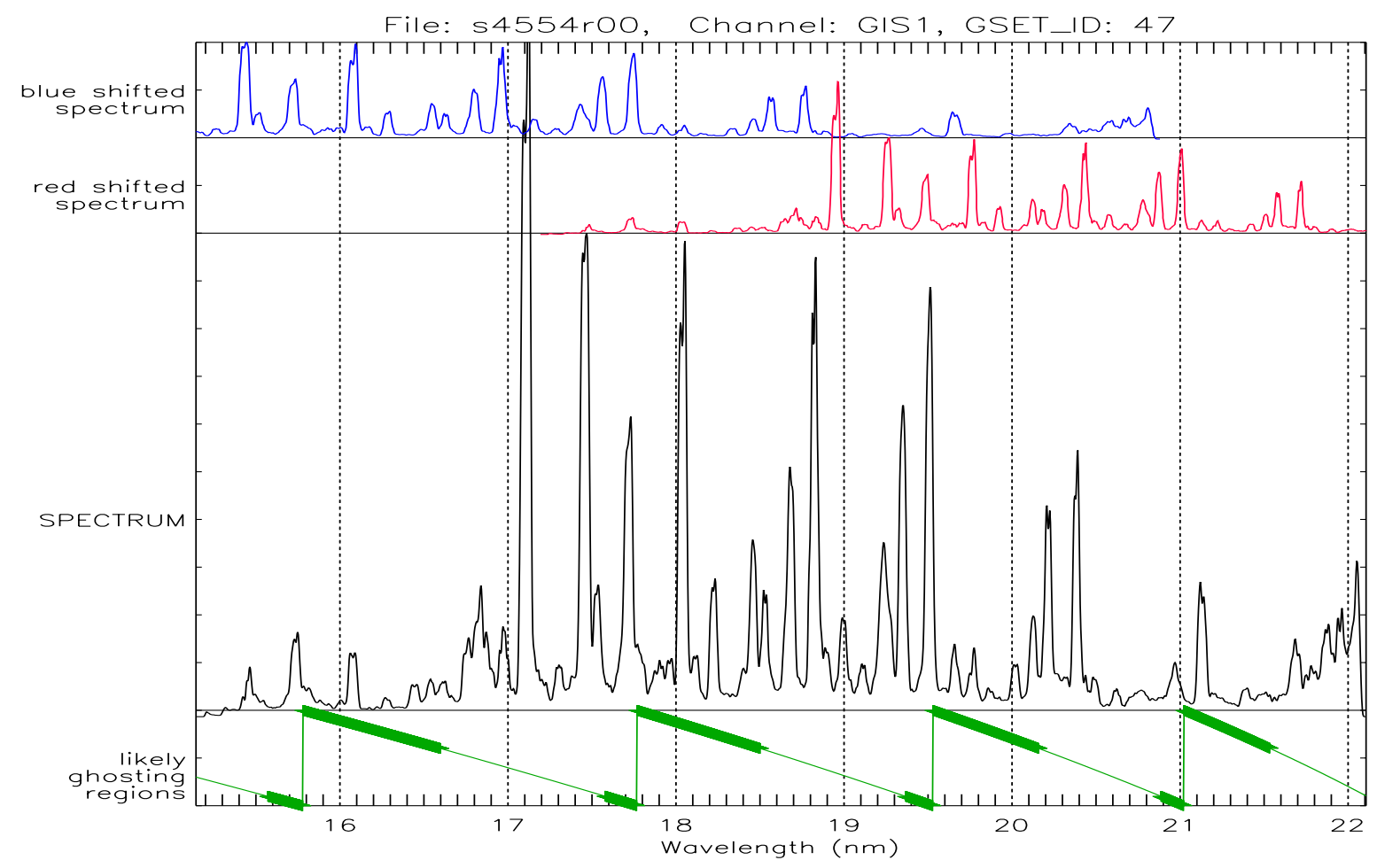

Fig. 3. Probable ghosting regions for the GIS 1 wavelength range. Similar examples are available in the CDS software for the other detectors. The main frame displays the observed (smoothed) spectrum; the top two panels display the blue-shifted and red-shifted spectrum. The bottom panel marks with a black line the regions where ghosts from the blue- and red-shifted spectra are likely to affect the observed spectrum

method is to try to relate each unidentified line position to the most probable ghosting line that could have produced it, with the assumption that the unidentified line is a ghost. If this assumption is correct it is possible to measure directly the shift between the ghost and its parent line; using several unidentified lines the ghosting line-ghost position relation could be determined. Using this empirical relationship it is possible to trace back the ghosts blending some identified line. Moreover, using the measured intensity of the unidentified lines a relationship between ghosted and parent intensities can be determined.

Using these methods it was possible to reconstruct the intensities of no more than a few lines, namely the lines reported in Fig. 4 and the Fe XIV $334.2 \AA$ and Fe XVI $335.4 \AA$ lines observed at the end of the GIS 2 detector, whose counts give rise respectively to the $317.6 \AA$ and $318.8 \AA$ lines. The paucity of the unambiguously identified and corrected ghosts compared to the great number of affected lines observed in the present dataset (more than half the total) is due to the complexity of the spectra themselves.

\subsection{Line fitting}

In order to measure line positions, widths and intensities it is necessary to use a fitting routine that adapts a known line profile to the observed spectral lines. In the present work use has been made of the ADAS fitting routines (Summers et al. 1996); full details can be found in Lang et al. (1990) and Brooks et al. (1998a). This routine is based on a maximum likelihood program which performs a multiple Gaussian fit together with a linear background.

The fit has been carried on leaving the background and line widths free to vary in order to better reproduce the observed spectrum.

The observed background emission results from a combination of true continuum emission, scattered light, detector effects, ghosts, contribution from weak emission lines. A proper evaluation of the background emission will be possible only when detector effects (some of which are present at the edges of the detectors) can be removed through flat-fielding. It should be noted that the background may be depleted by ghosting.

The ADAS program provides also the uncertainties of the fitted quantities. These include the uncertainties on the pre-flight sensitivities, as reported in Table 5 .

\subsection{Line widths}

Some comments are needed about line widths, since their values are very important for the measurement of line intensities. 

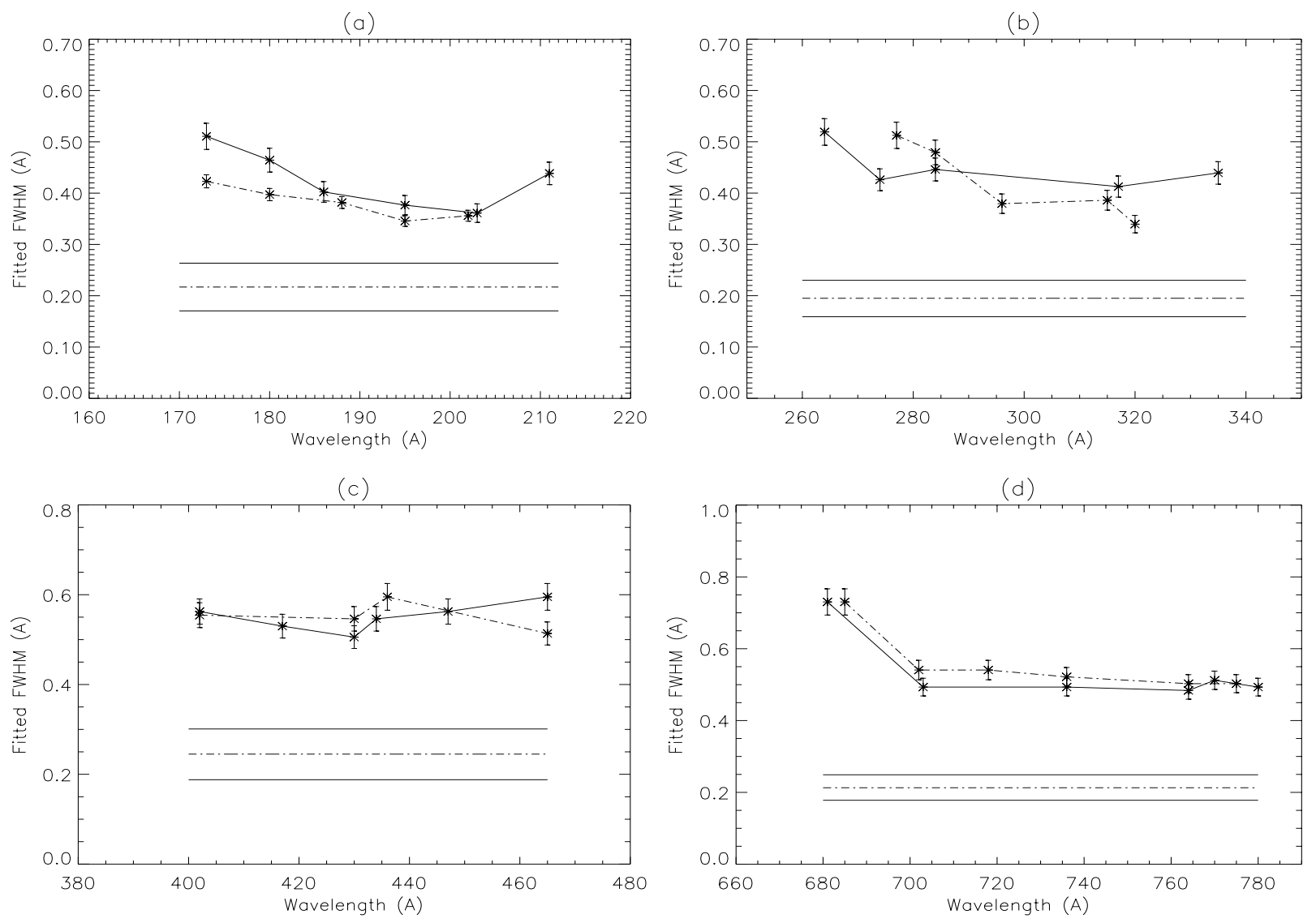

Fig. 5. Line widths for the GIS spectrometer, observed in quiet (dashed line) and active (full line) Sun conditions. The straight lines represent the mean of the pre-flight measured values and its experimental uncertainties. a) GIS 1 ; b) GIS 2; c) GIS 3; d) GIS 4

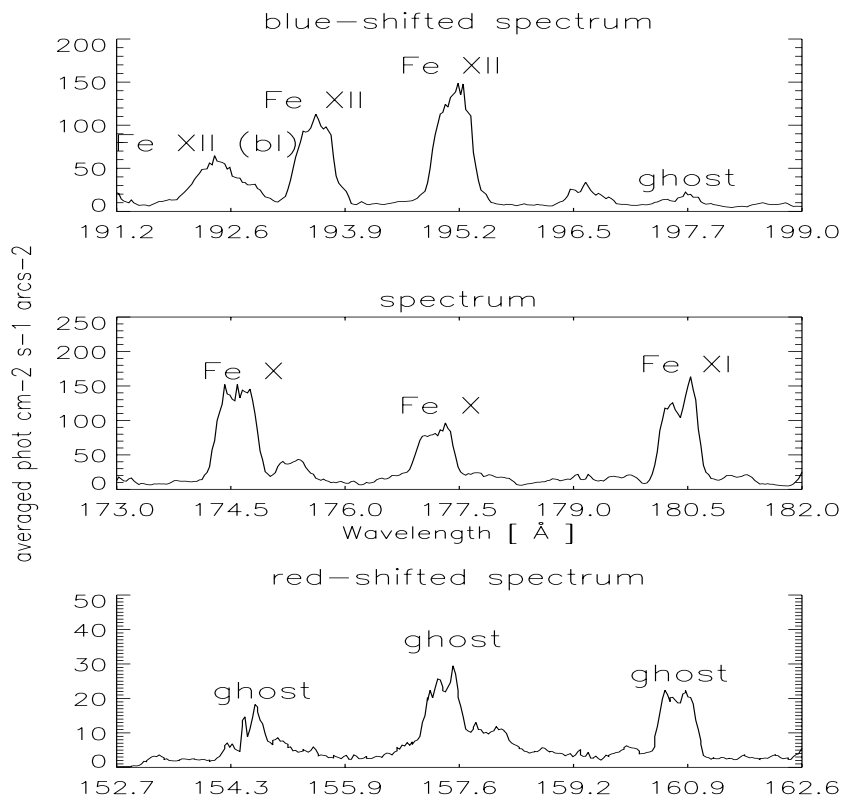

Fig. 4. Probable ghosting regions for the GIS 1 wavelength range. These show the corresponding spectral regions in the adjacent arms of the spiral detector pattern. Similar examples are available in the CDS software for the other detectors
In their calibration report, Bromage et al. (1996) have measured the line Full Width Half Maximum (FWHM) line widths for the four GIS detectors using a narrowbeam source of EUV radiation (Hollandt et al. 1994).

Some modulation of the FWHM along each detector was observed as expected, due to the configuration of the detectors along the Rowland Circle and to the variation of the point spread function and dispersion with wavelength. These values include broadening due to the limited width of the beam used.

The mean of the half-widths measured along each of the four GIS detectors is displayed in Fig. 5 (straight lines), together with their uncertainties.

The authors suggested that some broadening of the lines should occur in-flight (when the instrument aperture is fully illuminated), due to slight deviations of the light passing through the edges of the aperture; this increase was expected to be smaller than $15 \%$ of the provided pre-flight line FWHM for most lines. However, the effect may vary along the detector with some indication that the problem might be worse nearer the ends than the middle. 
In the present study the FWHM of the lines has been measured, using the adopted fitting program, for some very strong and isolated lines, where no blending effects were present and where no ghosting line was expected. The behaviour of the FWHM for all the four detectors for both active and quiet Sun has been investigated, and the results are displayed in Fig. 5. The uncertainties have been determined as statistical uncertainties of the fitting, and their values represent the estimated $95 \%$ confidence limit of the fitted quantity. The slit used in the observation is the $2^{\prime \prime} \times 2^{\prime \prime}$ slit. Different lines were used for the measurements in the two different spectra due to the lack of hot lines (such as Fe XIV, XV and XVI) in the quiet Sun spectrum.

These values have been compared to the FWHM measured in the pre-flight study by Bromage et al. (1996). It is important to note however that in the pre-flight study a narrow beam source has been used which did not fully illuminate the grating of the spectrometer. For this reason line widths were expected to be higher than in the case of full illuminated grating. This effect is greatest for GIS 4 (a 1.6 magnification factor) and smallest for GIS 1 (no magnification), and these factors are included in the pre-flight measured line widths reported by Bromage et al.

It is possible to see that in nearly all cases there are significant deviations from the pre-flight FWHM. In all cases the in-flight measurements of line width are greater than the preflight ones reported in Bromage et al. (1996). The differences between the two measurements range between a factor 1.4 to 2.4 , and present some slight variation between the four detectors. It is interesting to note that also Brooks et al. (1998a) find that their NIS in-flight FWHM measurements are greater than the pre-flight values, although the differences are somewhat smaller and range between a factor 1.1 to 2.2 . It was also noted that during ground calibration the spectral line positions moved when illuminating different parts of the GIS aperture. This optical effect is a suggested cause for the increase in original line profiles shown in Fig. 2a.

The differencies between the Bromage et al. (1996) laboratory mean FWHM and the values displayed in Fig. 5 are dependent on wavelength inside each channel. This wavelength dependence is consistent with pre-flight results.

\section{Results}

\subsection{The DEM analysis}

\subsubsection{Line selection}

The $D E M$ analysis requires the use of density insensitive lines for determining the Correction Function, so that the measurements of $\omega\left(T_{0}\right)$ are not dependent on $N_{\mathrm{e}}$. For this reason, we have used only density-insensitive or weakly density-sensitive lines. For the latter, theoretical intensities have been calculated assuming $N_{\mathrm{e}}=10^{9.4} \mathrm{~cm}^{-3}$ in active Sun and $N_{\mathrm{e}}=10^{9} \mathrm{~cm}^{-3}$ in quiet Sun; these values were determined from the data using the FexIII 203.80/202.04 line ratio.

The line selection for the $D E M$ study has been carried out with the additional requirement that line ghosts should not play a major role in the determination of the observed intensity of the candidate lines. For this reason all the weak lines which are likely to be blended with ghosts generated by strong lines have been rejected, and strong lines producing ghosts have been omitted from this study. There are a few exceptions to the latter requirement, for lines whose ghosts could be traced back and corrected and lines whose ghosts are too weak to alter significantly $(\leq 10 \%)$ the total intensity of the lines.

The DEM study allows the direct comparison of the intensities of lines emitted by different ions formed at similar $T_{\text {eff }}$ and observed in different GIS channels. The values of the $D E M$ provided by such lines are expected to be identical within experimental uncertainties, thus allowing a first check on the relative intensity calibration of the different GIS channels.

The lines used in the $D E M$ analysis are listed in Table 1, indicated by "dem" flag and ordered in temperature. The lines flagged with " $N e$ " are density-sensitive and can be used for estimating electron densities.

As Table 1 shows, several cool lines are present (mostly in the GIS 4 channel) and may be used in both quiet Sun and active region spectra, to constrain the $D E M$ for temperatures as low as $10^{4.6}$. Since opacity may play an important role in determining the observed intensities of some of the coolest lines used in the present study (N II, N III - Brooks et al. 1998b), the low temperature tail of the $D E M$ curve should always be treated with caution. Lines from N, O, Ne, Na, Mg, Al, Si, S and Fe were used for this study. This allowed a check on element abundances, in the sense that lines of different elements but emitted at the same temperature should have the same $D E M$ values, if the relative abundances applied were correct.

\subsubsection{Quiet Sun}

The presence of the hot Fexv line at $284.160 \AA$ ensures that the quiet Sun $D E M$ can be studied for temperatures as high as $210^{6} \mathrm{~K}$.

The resulting quiet Sun $D E M$ curve is displayed in Fig. 6 (top). The theoretical and experimental intensities are reported in Tables 2 and 3 .

The $\mathrm{O}, \mathrm{N}$ and Ne lines, observed in the GIS 3 and GIS 4 channels, are in relatively good agreement between themselves, with the only exception of the cool N II and O II lines, which may be affected by some opacity problem. The Ne IV multiplet agrees within $15 \%$ with N IV and $\mathrm{OV}$ lines formed at similar $T_{\text {eff }}$, as well as $\mathrm{Ne} \mathrm{v}$ and $\mathrm{OV}$, 
Table 1. Lines used for the analysis. $T_{\max }$ is the maximum abundance temperature of the ions. Lines marked with dem have been used for $D E M$ analysis, those marked with $\mathrm{Ne}$ can be used for density diagnostics. Where marked, these lines have proved to be reliable for analysis purposes and are recommended for further studies with GIS

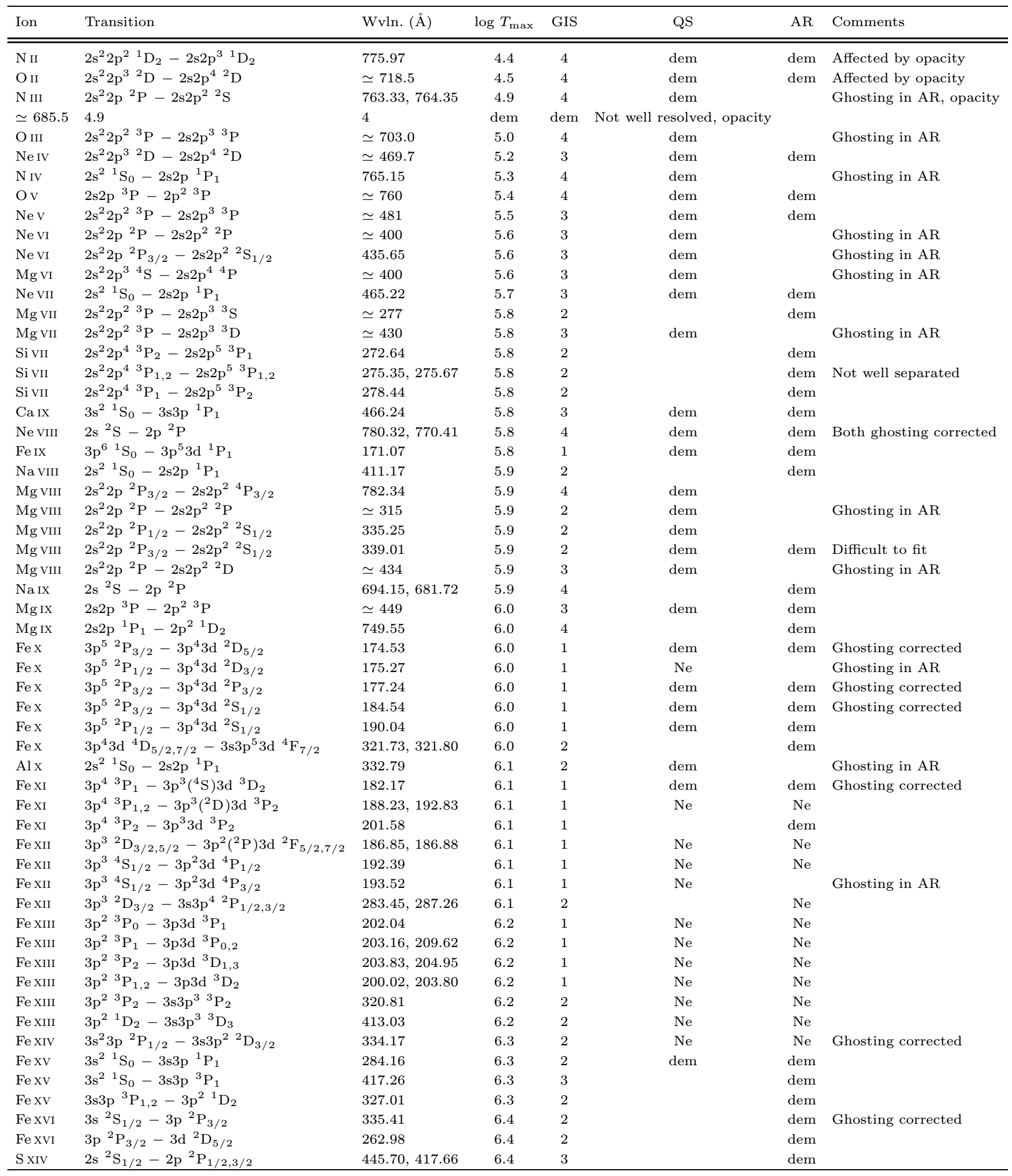


Table 2. Intensities of the lines used in the $D E M$ study (phot $\mathrm{cm}^{-2} \mathrm{~s}^{-1} \operatorname{arcsec}^{-2}$ ). Left) active region spectrum; right) quiet Sun spectrum. Theoretical intensities have been calculated with the derived $D E M$. Lines marked with * may have problems with ghosting. They have not been used for DEM diagnostics but have been reported in order to show the effects of ghosting on line intensities

\begin{tabular}{|c|c|c|c|c|c|c|c|c|c|}
\hline Ion & Wvl. (A) & GIS & $I_{\mathrm{ar}}$ & $\sigma_{\mathrm{ar}}$ & $I_{\mathrm{ar}}^{\mathrm{theor}}$ & $I_{\mathrm{qs}}$ & $\sigma_{\mathrm{qs}}$ & $I_{\mathrm{qs}}^{\text {theor }}$ & $T_{\max }$ \\
\hline $\mathrm{N}$ II & 775.965 & 4 & 22.6 & 3.9 & 6.9 & 4.0 & 0.8 & 2.5 & 4.4 \\
\hline O II & 718.610 & 4 & 34.4 & 5.8 & 34.6 & 13.4 & 2.3 & 18.9 & 4.5 \\
\hline N III & 763.333 & 4 & $8.5^{\star}$ & $1.8^{\star}$ & $5.0^{\star}$ & 1.9 & 0.6 & 3.0 & 4.9 \\
\hline N III & 764.351 & 4 & $9.7^{\star}$ & $1.9^{\star}$ & $9.6^{\star}$ & - & - & - & 4.9 \\
\hline O III & 702.332 & 4 & $17.7^{\star}$ & $3.0^{\star}$ & $20.4^{\star}$ & 7.7 & 1.3 & 9.9 & 5.0 \\
\hline O III & 702.897 & 4 & $52.7^{\star}$ & $10.0^{\star}$ & $60.3^{\star}$ & 27.2 & 4.6 & 29.4 & 5.0 \\
\hline O III & 703.854 & 4 & $83.3^{\star}$ & $14.2^{\star}$ & $103.0^{\star}$ & 42.6 & 7.2 & 50.1 & 5.0 \\
\hline Ne IV & 469.823 & 3 & 17.5 & 7.7 & 23.4 & 5.8 & 2.6 & 5.6 & 5.2 \\
\hline $\mathrm{N}$ IV & 765.146 & 4 & $122.5^{\star}$ & $20.8^{\star}$ & $114.0^{\star}$ & 55.9 & 9.5 & 43.8 & 5.3 \\
\hline $\mathrm{Ov}$ & 758.676 & 4 & 17.3 & 2.9 & 20.1 & 3.9 & 0.7 & 3.4 & 5.4 \\
\hline $\mathrm{Ov}$ & 759.441 & 4 & 15.6 & 2.7 & 15.2 & 3.0 & 0.6 & 2.6 & 5.4 \\
\hline $\mathrm{Ov}$ & 760.446 & 4 & 75.0 & 12.8 & 71.0 & 16.1 & 2.7 & 12.0 & 5.4 \\
\hline $\mathrm{Ov}$ & 762.003 & 4 & 22.1 & 3.8 & 18.8 & 2.7 & 0.5 & 3.2 & 5.4 \\
\hline $\mathrm{NeV}$ & 482.982 & 3 & 31.8 & 14.0 & 30.8 & 4.6 & 2.0 & 5.1 & 5.5 \\
\hline $\mathrm{Ne}$ VI & 401.136 & 3 & $42.0^{\star}$ & $18.5^{\star}$ & $36.6^{\star}$ & 7.2 & 3.2 & 6.7 & 5.6 \\
\hline $\mathrm{Ne}$ VI & 435.648 & 3 & $23.8^{\star}$ & $10.5^{\star}$ & $31.2^{\star}$ & 6.3 & 2.8 & 5.4 & 5.6 \\
\hline Mg VI & 399.281 & 3 & - & - & - & 1.6 & 0.7 & 2.8 & \\
\hline Mg VI & 400.666 & 3 & - & - & - & 4.5 & 2.0 & 5.3 & \\
\hline Ne VII & 465.220 & 3 & 407.7 & 179.4 & 335.0 & 78.6 & 34.6 & 59.5 & 5.7 \\
\hline Si VII & 272.638 & 2 & 44.3 & 5.8 & 52.0 & $3.1^{\star}$ & $0.4^{\star}$ & $3.1^{\star}$ & 5.8 \\
\hline Si VII & 275.353 & 2 & 144.8 & 18.8 & 222.0 & $9.3^{\star}$ & $1.2^{\star}$ & $13.8^{\star}$ & 5.8 \\
\hline Si VII & 278.443 & 3 & 154.9 & 20.1 & 210.6 & - & - & - & 5.8 \\
\hline Ca IX & 466.239 & 3 & 212.0 & 93.3 & 45.2 & 8.8 & 3.9 & 1.9 & 5.8 \\
\hline Mg VII & 276.154 & 3 & 34.3 & 4.5 & 28.4 & - & - & - & 5.8 \\
\hline Mg VII & 429.140 & 3 & $45.5^{\star}$ & $20.0^{\star}$ & $52.3^{\star}$ & 2.7 & 1.2 & 3.1 & 5.8 \\
\hline Mg VII & 431.313 & 3 & $127.0^{\star}$ & $55.9^{\star}$ & $155.0^{\star}$ & 6.9 & 3.0 & 7.3 & 5.8 \\
\hline Mg VII & 434.917 & 3 & $189.9^{\star}$ & $83.6^{\star}$ & $235.0^{\star}$ & 11.4 & 5.0 & 13.9 & 5.8 \\
\hline Ne VIII & 770.408 & 4 & 406.3 & 69.0 & 1030.0 & 72.8 & 12.4 & 151.0 & 5.8 \\
\hline Ne VIII & 780.323 & 4 & 232.6 & 39.5 & 520.0 & 46.7 & 7.9 & 76.4 & 5.8 \\
\hline Na VIII & 411.166 & 3 & 79.7 & 35.1 & 54.8 & - & - & - & 5.9 \\
\hline Fe IX & 171.073 & 1 & 3221.0 & 612.0 & 3230.0 & 260.1 & 49.4 & 214.0 & 5.8 \\
\hline
\end{tabular}

despite some difference in their $T_{\text {eff }}$. All this suggests that both the relative GIS 3 - GIS 4 intensity calibration and the abundances of these three elements are approximately correct.

Some problems arise between the aforementioned $\mathrm{N}$, $\mathrm{O}$ and $\mathrm{Ne}$ lines and $\mathrm{Mg}$ lines. $\mathrm{Mg}$ VI and $\mathrm{Mg}$ VII lines $T_{\text {eff }}$ values are very close to those of $\mathrm{NeV}$, VI and VII, but the $D E M$ value they provide always disagree, the $\mathrm{Mg}$ theoretical intensities being overestimated by a factor $\simeq 2.8$ relatively to the Neon values. Since the $\mathrm{Mg} \mathrm{VI}$, VII and Ne VI, VII lines are observed in the GIS 3 spectral range, any intercalibration problem is not expected. Moreover, the slight density-dependence of the Mg VI and VII lines is not able to account for the discrepancy. For these reasons, and remembering that the $\mathrm{O}, \mathrm{N}$ and $\mathrm{Ne}$ abundances are in agreement, the abundances of these three element were increased by a factor 3 in the $D E M$ analysis, bringing relative element abundances closer to photospheric values. It is worth noting that $\mathrm{N}, \mathrm{O}$ and $\mathrm{Ne}$ have high First Ionization Potential (FIP) while all the other elements used for the quiet Sun DEM study are low-FIP elements. The disagreement found between the Feldman (1992) coronal abundances and those required by the present dataset suggests a much reduced FIP effect (see Haisch et al. 1996) for this quiet Sun observation.

Ne VIII deserves some comment. The lines of this ion, observed in GIS 4, are formed at the same $T_{\text {eff }}$ as Mg VIII, and unlike the other Ne ions they are in relatively good agreement with the $\mathrm{Mg}$ lines with the Feldman (1992) abundances. The correction factor for the Ne, N and O elements required by the other Ne ions causes the Ne VIII lines to disagree with the other $\mathrm{Mg}$ lines by a similar 
Table 3. Intensities of the lines used in the $D E M$ study (phot $\mathrm{cm}^{-2} \mathrm{~s}^{-1} \operatorname{arcsec}^{-2}$ ). Left) active region spectrum; right) quiet Sun spectrum. Theoretical intensities have been calculated with the derived $D E M$. Lines marked with * may have problems with ghosting. They have not been used for DEM diagnostics but have been reported in order to show the effects of ghosting on line intensities

\begin{tabular}{|c|c|c|c|c|c|c|c|c|c|}
\hline Ion & Wvl. (̊) & GIS & $I_{\mathrm{ar}}$ & $\sigma_{\mathrm{ar}}$ & $I_{\mathrm{ar}}^{\text {theor }}$ & $I_{\mathrm{qs}}$ & $\sigma_{\mathrm{qs}}$ & $I_{\mathrm{qs}}^{\text {theor }}$ & $T_{\max }$ \\
\hline Mg VIII & 311.795 & 2 & - & - & - & 5.2 & 0.7 & 7.2 & 5.9 \\
\hline Mg VIII & 313.754 & 2 & - & - & - & 13.7 & 1.8 & 13.5 & 5.9 \\
\hline Mg VIII & 315.039 & 2 & - & - & - & 38.7 & 5.0 & 37.4 & 5.9 \\
\hline Mg VIII & 317.038 & 2 & - & - & - & 12.0 & 1.6 & 9.6 & 5.9 \\
\hline Mg VIII & 335.253 & 2 & - & - & - & 6.6 & 0.4 & 6.5 & 5.9 \\
\hline Mg VIII & 339.006 & 2 & 166.3 & 21.6 & 148.0 & - & - & - & 5.9 \\
\hline Mg VIII & 428.318 & 3 & $14.1^{\star}$ & $6.2^{\star}$ & $8.6^{\star}$ & - & - & - & 5.9 \\
\hline Mg VIII & 430.465 & 3 & $158.8^{\star}$ & $69.9^{\star}$ & $279.0^{\star}$ & 18.0 & 7.9 & 18.0 & 5.9 \\
\hline $\mathrm{Mg}$ VIII & 436.734 & 3 & $414.7^{\star}$ & $182.5^{\star}$ & $427.0^{\star}$ & 29.6 & 13.0 & 32.6 & 5.9 \\
\hline Mg VIII & 782.913 & 4 & - & - & - & 2.1 & 0.4 & 1.6 & 5.9 \\
\hline Na IX & 681.721 & 4 & 72.5 & 12.3 & 140.0 & - & - & - & 5.9 \\
\hline Na IX & 694.147 & 4 & 50.6 & 8.6 & 70.9 & - & - & - & 5.9 \\
\hline Mg IX & 439.176 & 3 & 47.4 & 20.9 & 26.8 & $2.6^{\star}$ & $1.1^{\star}$ & $1.6^{\star}$ & 6.0 \\
\hline Mg IX & 441.199 & 3 & 32.9 & 14.5 & 25.7 & $1.9^{\star}$ & $0.8^{\star}$ & $1.3^{\star}$ & 6.0 \\
\hline Mg IX & 448.293 & 3 & 39.0 & 17.2 & 25.2 & $2.1^{\star}$ & $0.9^{\star}$ & $1.6^{\star}$ & 6.0 \\
\hline Mg IX & 749.551 & 4 & 13.9 & 4.4 & 9.4 & - & - & - & 6.0 \\
\hline Fex & 174.534 & 1 & 2461.0 & 467.6 & 2790.0 & 237.7 & 45.2 & 197.0 & 6.0 \\
\hline Fex & 177.243 & 1 & 1646.0 & 312.7 & 1740.0 & 115.9 & 22.0 & 122.0 & 6.0 \\
\hline Fex & 184.543 & 1 & 745.7 & 141.7 & 743.0 & 54.2 & 10.3 & 51.6 & 6.0 \\
\hline Fex & 190.043 & 1 & 330.2 & 62.7 & 279.0 & 21.0 & 4.0 & 20.6 & 6.0 \\
\hline Fex & 321.731 & 2 & 29.2 & 14.0 & 39.1 & - & - & - & 6.0 \\
\hline $\mathrm{Alx}$ & 332.789 & 2 & $339.9^{\star}$ & $44.2^{\star}$ & $254.0^{\star}$ & 13.5 & 1.8 & 12.5 & 6.1 \\
\hline Fe XI & 182.168 & 1 & 568.7 & 108.1 & 511.0 & 20.2 & 3.8 & 25.0 & 6.1 \\
\hline Fe XI & 201.576 & 1 & 104.0 & 38.2 & 93.9 & - & - & - & 6.1 \\
\hline Fexv & 284.160 & 2 & 5574.0 & 724.6 & 7610.0 & 24.1 & 3.1 & 23.6 & 6.3 \\
\hline $\mathrm{Fexv}$ & 327.011 & 2 & 78.7 & 10.2 & 88.9 & - & - & - & 6.3 \\
\hline Fexv & 417.257 & 3 & 400.8 & 176.4 & 344.0 & - & - & - & 6.3 \\
\hline FexVI & 262.984 & 2 & 147.0 & 19.1 & 156.0 & - & - & - & 6.4 \\
\hline Fe XVI & 335.409 & 2 & 3917.0 & 509.2 & 2950.0 & - & - & - & 6.4 \\
\hline S XIV & 445.700 & 3 & 38.1 & 16.8 & 42.5 & - & - & - & 6.4 \\
\hline
\end{tabular}

amount; the cause for this peculiar behaviour is still not understood. Ghosts are unlikely to be the cause of this discrepancy since the Ne VIII ghosts can be traced back and corrected; possible intensity calibration problems between GIS 4 and the other channels are ruled out by the O, N and Ne lines. It is important to note that the same problem with Ne VIII is found in the active Sun DEM analysis, where no need to change the Feldman (1992) abundances is found (see Sect. 6.1.3).

$\mathrm{Mg}$ VIII and Mg IX lines have similar $T_{\text {eff }}$ to Fe IX, Fe X, $\mathrm{Si}$ VII and $\mathrm{Al} \mathrm{X}$ lines and the transitions from several ions of these elements are in good agreement. Since these ions are observed in the GIS 1, GIS 2 and GIS 3 channels, their agreement both confirms that the relative intensity calibration of these channels have no problems and that the adopted relative abundances of the $\mathrm{Mg}, \mathrm{Al}, \mathrm{Si}$ and $\mathrm{Fe}$ elements should be approximately correct.
It is also important to note that the $\mathrm{Mg}$ VIII intercombination transition at $782.34 \AA$ is in agreement with the other Mg VIII lines and this further suggests that the GIS 2, GIS 3 and GIS 4 relative intensity calibration is approximately correct.

\subsubsection{Active Sun}

The active region spectrum presented some relatively hot and strong lines (like FexV, FexVI and S XIV) which allowed a good determination of the $D E M$ curve for temperatures up to $10^{6.4} \mathrm{~K}$. The resulting active region $D E M$ is displayed in Fig. 6 (bottom). The theoretical and experimental intensities are reported in Tables 2 and 3.

In the present active region spectrum no evidence is found for a need to change the adopted element abundances. Most of the lines used for the active region $D E M$ 

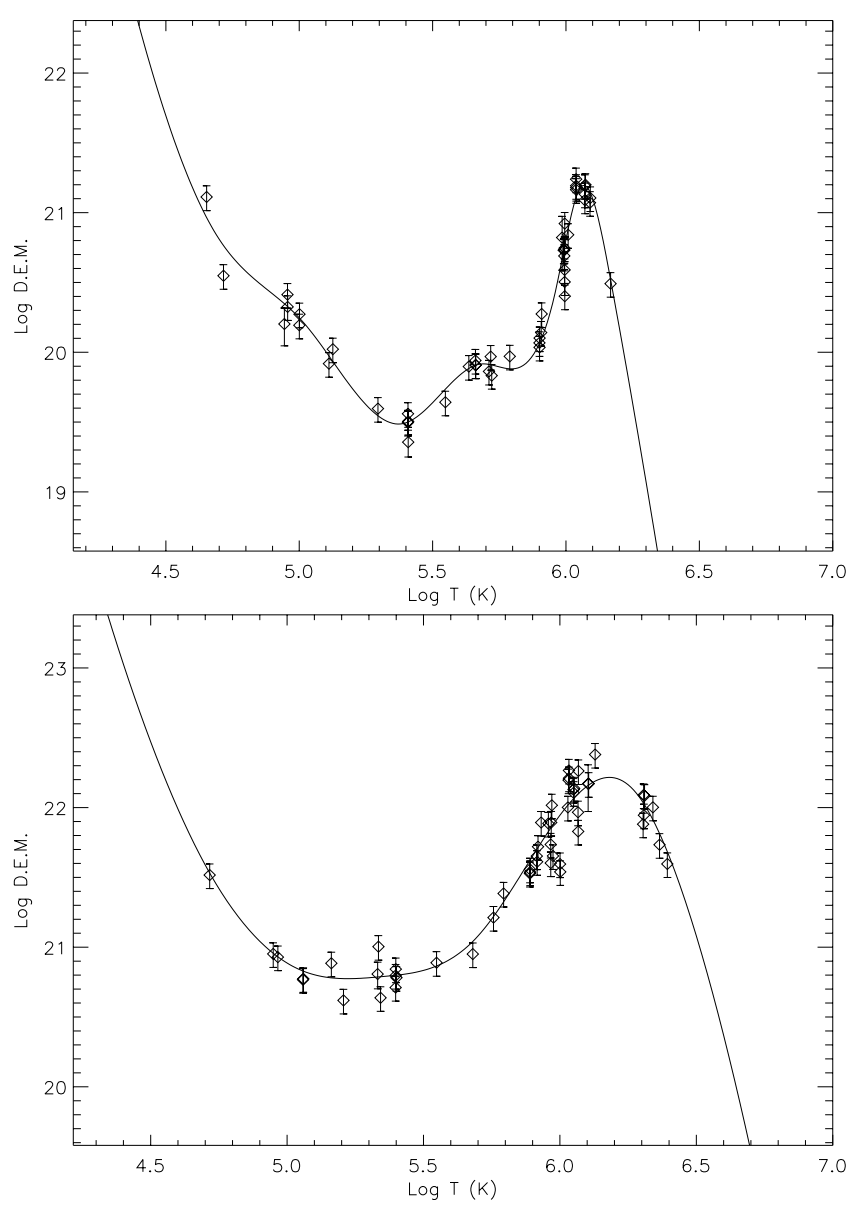

Fig. 6. Top) $D E M$ for the quiet Sun region: the mean $\chi^{2}$ is 3.6; bottom) $D E M$ for the active Sun region: the mean $\chi^{2}$ is 2.8

determination show agreement to better than $20 \%$, with only a few exceptions due to blending and uncertainties in the fitting of the background.

As in the quiet Sun spectrum, some more serious problems seem to arise with the GIS $4 \mathrm{Ne}$ VIII lines whose $T_{\text {eff }}$ is around $10^{6} \mathrm{~K}$. In spite of the agreement between $\mathrm{Ne}$ and $\mathrm{Mg}$ abundances the Ne VIII GIS 4 doublet observed at $770.4 \AA$ and $779.5 \AA$ never agree with the $\mathrm{Mg}$ VIII and Mg IX transitions, whose $T_{\text {eff }}$ is very similar to the Ne VIII values. The theoretical intensity of the Neon lines seem to be overestimated by a factor $\simeq 2.5$ relatively to those of the $\mathrm{Mg}$ lines - a factor very similar to the value found in the quiet Sun spectrum. These Ne VIII transitions have been corrected for ghosting. Ne VIII is observed in GIS 4, while the other $\mathrm{Mg}$ lines are observed in GIS 3, and this could suggest some problem in the relative calibration of the two channels. Nevertheless, the other lines observed in GIS 3 and GIS 4 agree within the errors (e.g. the Mg IX $749.55 \AA$ line with the other GIS $3 \mathrm{Mg}$ IX lines), indicating that no gross intercalibration correction is required between these two channels. Therefore the case of the Ne VIII active region lines is isolated, and does not provide any

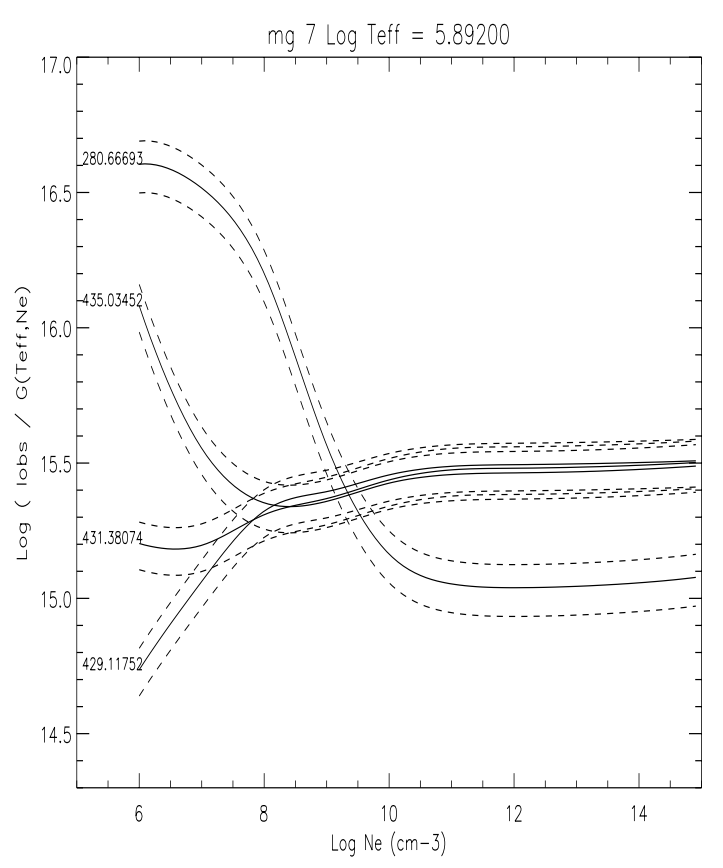

Fig. 7. L-functions for the Mg viI lines observed in the CDS GIS-2 and GIS-3 detectors. All the lines meet at $N_{\mathrm{e}} \simeq$ $10^{9.4} \mathrm{~cm}^{-3}$

definitive conclusion about the GIS 4 intensity calibration relative to the other channels.

The Fexv, XVI and SxIV lines, observed in GIS 1, GIS 2 and GIS 3, are in good agreement. This is a further indication that no correction is required to the GIS 1, GIS 2 and GIS 3 relative intensity calibration.

\subsection{GIS relative calibration}

The strongest evidence for any possible problems in the GIS relative calibration may be obtained through the analysis of lines emitted by the same ions and observed in more than one spectral window, since in this way any element abundance problem is ruled out, as well as problems due to variations in ion abundance. Also, no assumptions on the emission measure have to be made. In the GIS spectrum there are only few ions whose lines are observed in more than one detector which can be used in this work: Mg VII; Mg VIII; Mg IX; Fe X; Fe XII; Fe XIII and FexV.

The Mg VII active region L-functions are displayed in Fig. 7, showing that all the L-functions meet for $N_{\mathrm{e}} \simeq$ $10^{9.4} \mathrm{~cm}^{-3}$, in agreement with the density value obtained with the Fe XIII lines. As the observed lines come from the GIS 2 and GIS 3 detectors, this is an indication that their relative calibration needs no correction. The $\mathrm{Mg}$ VIII quiet Sun L-functions are displayed in Fig. 8, indicating that the electron density is between $10^{8}$ and $10^{12} \mathrm{~cm}^{-3}$, which is consistent with the value used in the $D E M$ analysis. An overall agreement is found between the lines, showing that all the displayed L-functions are equal within the 


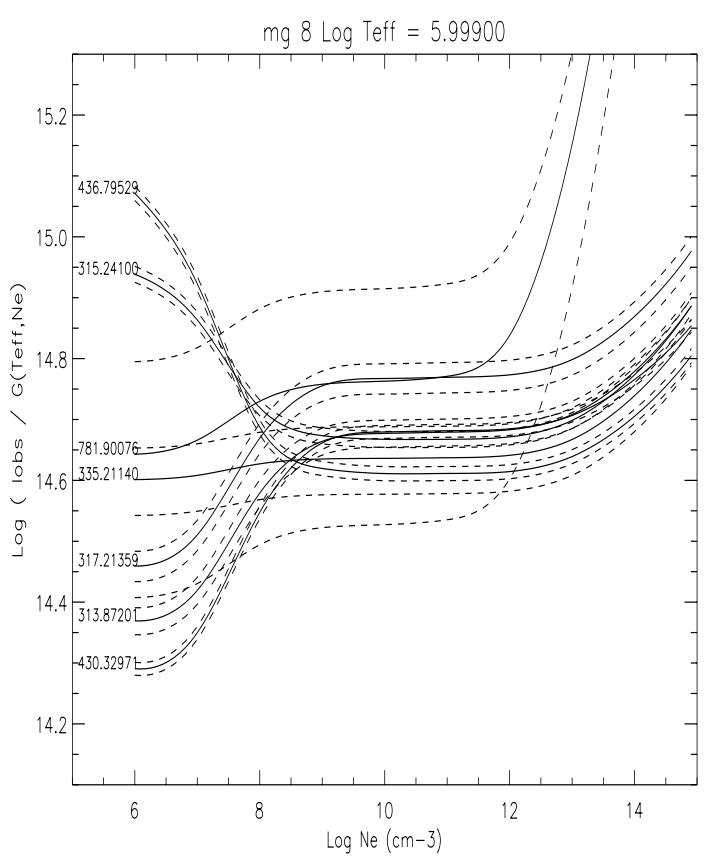

Fig. 8. Quiet Sun L-functions for the Mg VIII lines observed in the CDS GIS-2 and GIS-3 detectors

experimental uncertainties in the $10^{8}$ and $10^{12} \mathrm{~cm}^{-3}$ density range.

For each of these ions, the L-functions of most of the observed lines are in agreement among themselves better than $20 \%$. This means that no gross correction is required by the GIS neither between different detectors nor as a function of wavelength within each of the detectors. Disagreements between the few remaining lines can be explained by atomic physics problem or blending. No systematic trend was observed in these discrepancies which could point to some problem in the relative calibration of the four GIS detectors; moreover problems with these lines have already been reported in literature (Young et al. 1998 and references therein) and the similarity of our results with those of Young et al. (1998) is in turn a further indication that the GIS intensity calibration is approximately correct.

\subsection{Second order calibration}

Only the GIS 3 and GIS 4 channels presented second order spectral lines, with the GIS 3 second order bandpass partially overlapping GIS $1(196-246 \AA)$, and with GIS 4 overlapping a small part of the GIS 2 channel $(329-392 \AA)$. Since most of the second order lines visible in GIS 3 and GIS 4 belong to ions formed at high temperatures, the second order intensity calibration has been assessed using the active region spectrum, since in the quiet Sun very few second order lines are visible. It is important to note, however, that whenever observing hot plasma, the contribution of second order lines in the
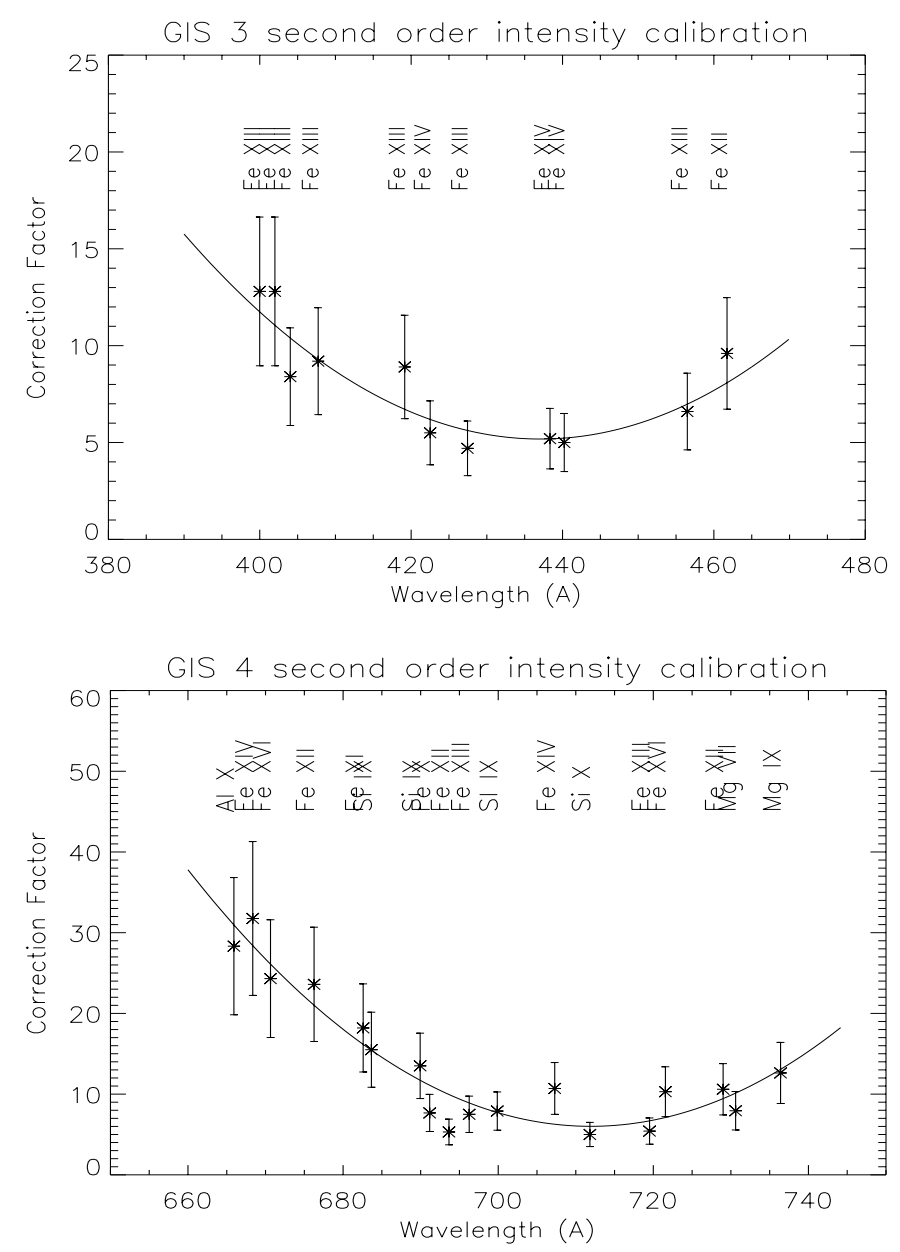

Fig. 9. Top) GIS 3 second order intensity calibration correction factor. Bottom) GIS 4 second order intensity calibration correction factor (see text)

two channels is non-negligible. In the pre-flight calibration (Bromage et al. 1996) no estimates of second order efficiencies were obtained.

Second order lines are sometimes either blended or affected by the ghosting problem and therefore only few of them can be used for intensity calibration studies with some confidence. The list of the lines used for second order calibration is given in Table 4 . Some of the GIS 3 and GIS 4 second order lines are observed also in GIS 1 and GIS 2 respectively and this allows a direct comparison of line intensities, and the measurement of the second to first order relative calibration is straightforward. In the remainder of the GIS 3 and GIS 4 spectral ranges measurements are made using the L-function method of line analysis described in Sect. 2.1.

In the present work we are concerned with the measurement of the correction factor $C$ so that the true intensity of second order lines can be obtained from the calibrated intensity $I_{\text {cal }}$ (calculated using the first order efficiencies) as

$I_{2^{\text {nd }}}=C \times I_{\text {cal }}$ 
Table 4. Lines used for GIS 3 (top) and GIS 4 (bottom) second-to-first order relative intensity calibration. Wavelengths are measured in $\AA$

\begin{tabular}{lrr}
\hline Ion & $\lambda_{\text {lab }}$ & $\lambda_{\text {obs }}$ \\
\hline \hline Fe XIII & 200.02 & 399.99 \\
Fe XIII & 201.13 & 402.00 \\
Fe XIII & 202.04 & 404.03 \\
Fe XIII & 203.80 & 407.72 \\
& 203.83 & \\
Fe XIII & 209.62 & 419.15 \\
Fe XIV & 211.30 & 422.52 \\
FeXIII & 213.77 & 427.45 \\
FeXIV & 219.14 & 438.35 \\
Fe XIV & 220.09 & 440.23 \\
FeXIII & 228.16 & 456.49 \\
Fe XII & 230.77 & 461.74 \\
\hline \hline AlX & 332.79 & 665.92 \\
Fe XIV & 334.17 & 668.35 \\
Fe XVI & 335.41 & 670.64 \\
Fe XII & 338.28 & 676.26 \\
FeXI & 341.11 & 682.59 \\
Si IX & 341.95 & 683.65 \\
Si IX & 344.95 & 689.94 \\
& 345.12 & \\
FeX & 345.72 & 691.16 \\
Fe XII & 346.85 & 693.65 \\
Fe XIII & 348.18 & 696.26 \\
Si IX & 349.79 & 699.88 \\
Fe XIV & 353.83 & 707.29 \\
Si X & 356.01 & 711.82 \\
& 356.05 & \\
Fe XIII & 359.64 & 719.51 \\
& 359.84 & \\
FeXVI & 360.76 & 721.56 \\
Fe XII & 364.47 & 729.00 \\
Mg VII & 365.18 & 730.61 \\
& 365.23 & \\
Mg IX & 365.24 & \\
\hline & & \\
& & \\
&
\end{tabular}

Error bars include the uncertainties in the atomic physics applied in the L-function technique.

\subsubsection{GIS 3 second order calibration}

The resulting correction curve has been fitted with a best fit second order polynomial:

$$
\begin{gathered}
C=a+b \lambda+c \lambda^{2} \\
a=1092.43 ; \quad b=-4.99092 ; \quad c=5.7266710^{-3}
\end{gathered}
$$

and can be applied to second order lines between $400 \AA$ and $465 \AA$. This GIS 3 second order calibration curve is displayed in Fig. 9 (top).
Table 5. Mean sensitivies of the Grazing Incidence Spectrometer (counts per photon)

\begin{tabular}{cc}
\hline Detector & Mean sensitivity \\
\hline \hline GIS 1 & $(2.1 \pm 0.4) 10^{-4}$ \\
GIS 2 & $(2.4 \pm 0.3) 10^{-4}$ \\
GIS 3 & $(3.4 \pm 1.5) 10^{-4}$ \\
GIS 4 & $(1.2 \pm 0.2) 10^{-4}$ \\
\hline
\end{tabular}

\subsubsection{GIS 4 second order calibration}

The resulting GIS 4 second order calibration curve is displayed in Fig. 9 (bottom). The correction curve has been fitted with a best fit second order polynomial:

$$
\begin{gathered}
C=a+b \lambda+c \lambda^{2} \\
a=5976.44 ; \quad b=-16.7723 ; \quad c=1.1779310^{-2}
\end{gathered}
$$

and can be applied to second order lines between $665 \AA$ and $740 \AA$.

\section{Conclusions and further work}

In this work the intensity calibration of the CDS Grazing Incidence Spectrometer is discussed, and plasma diagnostic techniques have been used to check the pre-flight relative intensity calibration of the four GIS detectors and for determining the second-to-first order relative intensity calibration of the GIS 3 and GIS 4 detectors.

A preliminary study has been performed in order to determine the effect of the ghosting problem on some observed emission lines, and to correct for it, whenever possible. This allowed the selection of a number of lines (Table 1) which have proved not to be significantly affected by the ghosting problem.

These lines have been used for checking the relative intensity calibration between the four detectors of the GIS instrument. A general agreement is found between the present results and the pre-flight intensity calibration by Bromage et al. (1996) listed in Table 5. Therefore the use of the pre-flight calibration values is recommended for intensity calibration of GIS spectra. No evidence was found for a wavelength dependent variation of sensitivity along the detectors.

For the first time, a second order sensitivity calibration has been determined. Correction factors have been obtained which may be applied to the first order sensitivities of GIS 3 and GIS 4, where nearly all the second order lines appear.

The accuracy of the present check of the GIS sensitivity is mainly limited by instrumental problems, such as fixed patterning, ghosting and anomalous line profiles, and by the uncertanties of the theoretical data used for the analysis. Most of the GIS lines which have proved to be 
useful for the present work agree among themselves within $30 \%$. The higher limit is mainly due to weaker lines. We consider this higher limit as the accuracy of the sensitivity calibration of the GIS instrument determined in the present work.

The problems of ghosting, fixed patterning and instrumental line profiles have been discussed, and several methods for smoothing the fixed patterning were compared. A 3 -point boxcar smoothing was found to be adequate.

Lines in Table 1 are recommended for further diagnostic studies of active region and quiet Sun spectra observed with GIS. However, it is important to be aware that the spectra analysed had GIS Setup IDs of 41 (quiet Sun) and GSET_ID $=47$ (active region), and therefore the ghosting analysis that allowed the selection of usable lines can in principle only be applied to other observations having the same GSET_ID. The position and amount of ghosting is not expected to vary for a given GSET_ID. What varies is the degree of ghosting, which can change drastically depending on the target source.

Any application of the presented results, in terms of ghost analysis, to other observations taken with a different GSET_ID should be treated with great caution. Also, any long term gain depression could modify the relative efficiencies of the GIS detectors, and therefore any application of the pre-flight calibration to observations taken before or after September 1996 should be checked. However, current indications are that detector sensitivities are stable with time.

There is much work still being done, both with the atomic physics and the GIS detector calibrations. These include software for refining and semi-automating the ghost detection and correction, second order calibrations for long term gain depression, as well as work with the other valid GSET_IDs.

Acknowledgements. G. Del Zanna work has been supported by a scholarship from the University of Central Lancashire. G. Del Zanna and B.J.I. Bromage are grateful for the use of PPARC Starlink computing facilities. We are grateful to all the SOHO and in particular CDS team members who have made this solar mission such a success. SOHO is a ESA and NASA joint mission. We are grateful to Dr. J.S. Kaastra for useful comments on the original manuscript.

\section{References}

Andretta V., Jones H.P., 1997, ApJ 489, 375

Arnaud M., Raymond J.C., 1992, ApJ 398, 394

Arnaud M., Rothenflug R., 1985, A\&AS 60, 425

Binello A.M., Mason H.E., Storey P.J., 1997, Adv. Sp. Res. 20, 2263

Binello A.M., Mason H.E., Storey P.J., 1998, A\&AS 127, 545

Breeveld A.A., 1996, Ultraviolet Detectors for Solar Observations on the SOHO Spacecraft, University of London Ph.D. Thesis

Breeveld A.A., Edgar M.L., Smith A., Lappington J.S., Thomas P.D., 1992, Rev. Sci. Instr. 63, 1

Bromage B.J.I., Breeveld A.A., Kent B.J., Pike C.D., Harrison R.A., 1996, UCLan Report CFA/96/09

Brooks D.H., et al., 1998a, A\&A (submitted)

Brooks D.H., et al., 1998b, A\&A (submitted)

Dere K.P., Landi E., Mason H.E., Monsignori Fossi B.C., Young P.R., 1997, A\&AS (in press)

Feldman U., 1992, Phys. Scr. 46, 202

Haisch B., Saba J.L.R., Meyer J.P., 1996, in: Astrophysics in the Extreme Ultraviolet, Bowyer S. and Malina R. (eds.), p. 511

Harrison R.A., et al., 1995, Sol. Phys. 162, 233

Haugan S.V.H., 1997, CDS software note No. 47, version 1

Hollandt J., et al., 1994, Appl. Opt., 33, 68

Landi E., Landini M., 1997, A\&A 327, 1230

Landi E., Landini M., 1998a, The Arcetri Spectral Code, A\&A (in press)

Landi E., Landini M., 1998b, Temperature and density diagnostics of quiet Sun and active regions observed with CDS NIS on SOHO, A\&A (submitted)

Landi E., Landini M., Pike C.D., Mason H.E., 1997, Sol. Phys. 175,553

Landini M., Monsignori Fossi B.C, 1991, A\&AS 91, 183

Lang J., Mason H.E., McWhirter R.W.P., 1990, Sol. Phys. 129, 31

Storey P.J., Mason H.E., Saraph H.E., 1998 (private communications)

Summers H.P., Brooks D.H., Hammond T.J., Lanzafame A.C., Lang J., 1996, RAL Technical Report RAL-TR-96-017, March 1996

Young P.R., Mason H.E., 1997, Sol. Phys. 175, 523

Young P.R., Landi E., Thomas R.J., 1998, A\&A 329, 291 